\title{
A CHARACTERIZATION OF BOUNDED SYMMETRIC DOMAINS BY CURVATURE
}

BY

\author{
J. E. D'ATRI AND I. DOTTI MIATELLO
}

\begin{abstract}
This paper will prove that a bounded homogeneous domain is symmetric if and only if, in the Bergman metric, all sectional curvatures are nonpositive.
\end{abstract}

Introduction. It is well known that a bounded symmetric domain has nonpositive sectional curvature in the Bergman metric. This paper is devoted to the converse, namely, that a bounded homogeneous domain (equivalently, homogeneous Siegel domain) which has nonpositive sectional curvature in the Bergman metric must be a symmetric domain. The proof uses the techniques of normal $j$-algebras [13], as well as results of Vinberg [16, 17], the first author [1-3], and, indirectly, Dorfmeister $[5,6]$. The body of the paper is in two sections. In the first, we derive certain relations between the dimensions of the root spaces in an irreducible normal $j$-algebra, which, in the presence of the additional curvature assumption, show the equality of the dimensions of all root spaces corresponding to certain roots for which no multiple is a root and the equality of the dimensions of all root spaces corresponding to roots which are half of another root. In the second section, we show that the first dimension result implies that the cone of the corresponding Siegel domain is self-dual while the two dimension results together imply that the domain is quasi-symmetric. The proof is then finished because [3] a quasi-symmetric domain with nonpositive sectional curvature in the Bergman metric is known to be symmetric. We remark that some of the subsidiary results mentioned above are not essentially new. However, for consistency of presentation, it is necessary to have them in the language of normal $j$ algebras and for that reason they are included. There is also overlap here with work of Zelow (Lundquist) [21].

1. Throughout this paper, $(\underline{s}, j)$ will denote a normal $j$-algebra with admissible form $\omega$. This means that $\underline{s}$ is a finite dimensional real split solvable Lie algebra with almost complex structure $j: \underline{s} \rightarrow \underline{s}$ such that $[X, Y]+j[j X, Y]+j[X, j Y]=[j X, j Y]$ and $\omega$ is a linear form on $\underline{s}$ such that the bilinear form $\langle X, Y\rangle=\omega[j X, Y]$ is symmetric, positive-definite, and $j$-invariant (note that the assumption that $\underline{s}$ is real split was incorrectly omitted in [2]). Let $\underline{n}=[\underline{s}, \underline{s}]$ and let $\underline{a}$ be the orthogonal complement of $\underline{n}$ in $\underline{s}$. By the basic structure theorem of Pyatetskii-Shapiro [13], $\underline{a}$ is a commutative

Received by the editors July 13, 1981.

1980 Mathematics Subiect Classification. Primary 32M10, 53C30.

(c) 1983 American Mathematical Society (c) 0002-9947/82/000-0759/\$09.00 
subalgebra and $n$ can be written as the orthogonal (cf. [1]) direct sum of the root spaces $\underline{n}_{\alpha}=\{X \in \underline{n}:[H, X]=\alpha(H) X, H \in \underline{a}\}$. If $\varepsilon_{1}, \ldots, \varepsilon_{R}$ are the roots whose root spaces are mapped into $\underline{a}$ by $j$, then $R=\operatorname{dim} \underline{a}$, the roots $\varepsilon_{1}, \ldots, \varepsilon_{R}$ are linearly independent, and, with proper labelling, all roots are of the form $\frac{1}{2} \varepsilon_{k}, \varepsilon_{k}, 1 \leq k \leq R$; $\frac{1}{2}\left(\varepsilon_{m} \pm \varepsilon_{n}\right), 1 \leq m<n \leq R$. Further, $j \underline{n}_{\varepsilon_{k} / 2}=\underline{n}_{\varepsilon_{k} / 2}$ and $j \underline{n}_{\left(\varepsilon_{m}+\varepsilon_{n}\right) / 2}=\underline{n}_{\left(\varepsilon_{m}-\varepsilon_{n}\right) / 2}, m<n$ (note the misprint on this relation in [2]). Since each root space $\underline{n}_{\varepsilon_{k}}$ is one dimensional, we can once and for all fix $X_{k} \in \underline{n}_{\varepsilon_{k}}$ so that $\varepsilon_{k}\left(j X_{l}\right)=\delta_{k l}$. We also need the following nondegeneracy condition, proved as part of the structure theorem by PyatetskiiShapiro [13].

(1) $[X, Z]$ is nonzero for nonzero $X \in \underline{n}_{\left(\varepsilon_{k}-\varepsilon_{l}\right) / 2}$ and nonzero

$$
Z \in \underline{n}_{\varepsilon_{l} / 2}+\sum_{s>l} \underline{n}_{\left(\varepsilon_{l}-\varepsilon_{s}\right) / 2}
$$

If $S$ is a connected, simply-connected, Lie group with Lie algebra $\underline{s}$, then $\langle$, induces a left invariant Riemannian metric (also denoted $\langle$,$\rangle ) on S$ which is Kähler with respect to the left invariant complex structure induced by $j$. The associated LeviCivita connection $\nabla$ is computed by

(2) $2\left\langle\nabla_{X} Y, Z\right\rangle=\langle[X, Y], Z\rangle+\langle[Z, X], Y\rangle+\langle[Z, Y], X\rangle, \quad X, Y, Z \in \underline{s}$.

In computing sectional curvatures, we will use

$$
\begin{aligned}
\langle R(Y, Z) Z, Y\rangle= & -\left\langle\nabla_{Z} Z, \nabla_{Y} Y\right\rangle+\left\langle\nabla_{Y} Z, \nabla_{Z} Y\right\rangle \\
& -\left\langle\nabla_{[Y, Z]} Z, Y\right\rangle, \quad Y, Z \in \underline{s} .
\end{aligned}
$$

Proposition 1. Let $(\underline{s}, j)$ be a normal j-algebra.

(1.1) If $\underline{n}_{\left(\varepsilon_{k}-\varepsilon_{l}\right) / 2} \neq 0$, then $\operatorname{dim} \underline{n}_{\varepsilon_{l} / 2} \leq \operatorname{dim} \underline{n}_{\varepsilon_{k} / 2}$ for $k<l$ and $\operatorname{dim} \underline{n}_{\left(\varepsilon_{l}-\varepsilon_{m}\right) / 2} \leq$ $\operatorname{dim} \underline{n}_{\left(\varepsilon_{k}-\varepsilon_{m}\right) / 2}$ for $k<l<m$.

(1.2) If $\underline{n}_{\left(\varepsilon_{l}-\varepsilon_{m}\right) / 2} \neq 0$, then $\operatorname{dim} \underline{n}_{\left(\varepsilon_{k}-\varepsilon_{l}\right) / 2} \leq \operatorname{dim} \underline{n}_{\left(\varepsilon_{k}-\varepsilon_{m}\right) / 2}$ for $k<l<m$. If in addition, the sectional curvature of the left invariant metric induced by $\langle$,$\rangle is nonpositive,$ then here we have equality of dimensions.

Proof. The inequalities are easy applications of (1). For example, (1.1) follows by picking any nonzero $X \in \underline{n}_{\left(\varepsilon_{k}-\varepsilon_{l}\right) / 2}$ and considering the injections

$$
\begin{aligned}
& \text { ad } X: \underline{n}_{\varepsilon_{l} / 2} \rightarrow \underline{n}_{\varepsilon_{k} / 2}, \\
& \text { ad } X: \underline{n}_{\left(\varepsilon_{l}-\varepsilon_{m}\right) / 2} \rightarrow \underline{n}_{\left(\varepsilon_{k}-\varepsilon_{m}\right) / 2}
\end{aligned}
$$

and the inequality in (1.2) follows by picking any nonzero $Z \in \underline{n}_{\left(\varepsilon_{l}-\varepsilon_{m}\right) / 2}$ and considering the injection

$$
\text { ad } Z: \underline{n}_{\left(\varepsilon_{k}-\varepsilon_{l}\right) / 2} \rightarrow \underline{n}_{\left(\varepsilon_{k}-\varepsilon_{m}\right) / 2}
$$

To prove equality in (1.2), consider any nonzero $Y \in \underline{n}_{\left(\varepsilon_{k}+\varepsilon_{m}\right) / 2}$ and compute the sectional curvature determined by $Y$ and $Z$ using (3). Note that expressions such as $[[Y, Z], Z]$ and $[Y,[Y, Z]]$ vanish because they would lie in root spaces corresponding 
to unallowed roots. Computing covariant derivatives from (2), one has

$$
\begin{aligned}
2\left\langle\nabla_{[Y, Z]} Z, Y\right\rangle & =\langle[[Y, Z], Z], Y\rangle+\langle[Y,[Y, Z]], Z\rangle+\langle[Y, Z],[Y, Z]\rangle \\
& =\|[Y, Z]\|^{2}, \\
\left\langle\nabla_{Y} Z, \nabla_{Z} Y\right\rangle & =\left\langle\nabla_{Y} Z, \nabla_{Y} Z+[Z, Y]\right\rangle \\
& =\left\|\nabla_{Y} Z\right\|^{2}+\frac{1}{2}(\langle[Y, Z],[Z, Y]\rangle+\langle[[Z, Y], Y], Z\rangle+\langle[[Z, Y], Z], Y\rangle) \\
& =\left\|\nabla_{Y} Z\right\|^{2}-\frac{1}{2}\|[Y, Z]\|^{2} .
\end{aligned}
$$

Finally, as in [1], for any root $\alpha$, we may define $H_{\alpha} \in \underline{a}$ so that $\left\langle H_{\alpha}, H\right\rangle=\alpha(H)$, $H \in \underline{a}$. Then for any $V \in \underline{n}_{\alpha},(2)$ implies $\nabla_{V} V=\langle V, V\rangle H_{\alpha}$. Further,

$$
H_{\varepsilon_{k}}=\frac{j X_{k}}{\omega\left(X_{k}\right)}, \quad\left\langle X_{k}, X_{k}\right\rangle=\omega\left(X_{k}\right)
$$

Thus

$$
\begin{aligned}
\left\langle\nabla_{Y} Y, \nabla_{Z} Z\right\rangle & =\frac{1}{4}\langle Y, Y\rangle\langle Z, Z\rangle\left\langle H_{\varepsilon_{k}+\varepsilon_{m}}, H_{\varepsilon_{l}-\varepsilon_{m}}\right\rangle \\
& =-\frac{1}{4}\|Y\|^{2}\|Z\|^{2} \frac{1}{\omega\left(X_{m}\right)} .
\end{aligned}
$$

Using (3), we get

$$
\langle R(Y, Z) Z, Y\rangle=\frac{1}{4}\|Y\|^{2}\|Z\|^{2} \frac{1}{\omega\left(X_{m}\right)}+\left\|\nabla_{Y} Z\right\|^{2}-\|[Y, Z]\|^{2} .
$$

Assuming nonpositive sectional curvature, we have $[Y, Z] \neq 0$. Thus we have the injection

$$
\operatorname{ad} Z: \underline{n}_{\left(\varepsilon_{k}+\varepsilon_{m}\right) / 2} \rightarrow \underline{n}_{\left(\varepsilon_{k}+\varepsilon_{l}\right) / 2}
$$

and

$$
\operatorname{dim} \underline{n}_{\left(\varepsilon_{k}-\varepsilon_{m}\right) / 2}=\operatorname{dim} \underline{n}_{\left(\varepsilon_{k}+\varepsilon_{m}\right) / 2} \leq \operatorname{dim} \underline{n}_{\left(\varepsilon_{k}+\varepsilon_{l}\right) / 2}=\operatorname{dim} \underline{n}_{\left(\varepsilon_{k}-\varepsilon_{l}\right) / 2} .
$$

REMARK. With the notation of the preceeding proposition, it is easy to see that $\nabla_{Y} Z$ must be in $\underline{n}_{\left(\varepsilon_{k}+\varepsilon_{l}\right) / 2}$ from which it follows that $\nabla_{Y} Z=\frac{1}{2}[Y, Z]$. Thus we have the relation

$$
\langle R(Y, Z) Z, Y\rangle=\frac{1}{4}\|Y\|^{2}\|Z\|^{2} \frac{1}{\omega\left(X_{m}\right)}-\frac{3}{4}\|[Y, Z]\|^{2}
$$

which is interesting since the norms involved depend only on $\omega\left(X_{k}\right)$ and $\omega\left(X_{l}\right)$.

We now introduce notation which will be used throughout this paper, namely

$$
n_{k l}=\operatorname{dim} \underline{n}_{\left(\varepsilon_{k}-\varepsilon_{l}\right) / 2}, \quad n_{k}=\operatorname{dim} \underline{n}_{\varepsilon_{k} / 2}
$$

We emphasize that the dimensions here are over the reals and we allow the possibility that some of these dimensions are zero. Also, we must recall two results proved in $[1,2]$ : 
(5) If $\frac{1}{2}\left(\varepsilon_{k}+\varepsilon_{l}\right)$ is a root (i.e., $n_{k l} \neq 0$ ) for some pair of indices $k<l$ and the sectional curvature of the left invariant metric induced by $\langle$,$\rangle is nonpositive, then$ $\omega\left(X_{k}\right) \leq \omega\left(X_{l}\right)$.

(6) The metric induced by $\langle$, $\rangle$ is Einstein if and only if

$$
\omega\left(X_{m}\right)=c\left(1+\frac{1}{4} n_{m}+\frac{1}{2} \sum_{l<m} n_{l m}+\frac{1}{2} \sum_{n>m} n_{m n}\right)
$$

for some positive constant $c$.

Note that the Bergman metric on a bounded homogeneous domain (equivalently, homogeneous Siegel domain) is Einstein and by results of Pyatetskii-Shapiro and Vinberg, every bounded homogeneous domain with Bergman metric will be holomorphically isometric to a connected, simply-connected, split solvable Lie group $S$ with left invariant complex structure and metric of the type we are considering. Hence the Bergman metric will be equivalent to a metric on $S$ defined by the equation in (6).

From now on, it will be convenient to assume that the domains we consider are irreducible. Since properties such as being symmetric or having nonpositive sectional curvature in the Bergman metric hold for a domain if and only if they hold for each irreducible factor, this is not a real restriction. If the domain is irreducible, then it is easy to see that the corresponding normal $j$-algebra is also irreducible in the sense that it cannot be decomposed as a sum of $j$-invariant ideals. It is in this form that the irreducibility assumption will be used.

Proposition 2. Let $(\underline{s}, j)$ be an irreducible normal j-algebra and suppose the metric induced by $\langle$,$\rangle is Einstein with nonpositive sectional curvature. Then there are con-$ stants $a$ and $b$ such that

$$
n_{i}=a, \quad 1 \leq i \leq R ; \quad n_{k l}=b, \quad 1 \leq k<l \leq R .
$$

In particular, $\omega\left(X_{i}\right)$ is a constant, independent of $i=1, \ldots, R$.

Proof. The case $R=1$ is trivial so assume $R>1$.

We will first show that if $n_{i R}=0$ for some $i=1, \ldots, R-1$, then $\underline{s}$ is reducible, contrary to assumption. Clearly, if $n_{i R}=0$ for all $i<R$, then $\underline{s}$ would decompose into two $j$-invariant ideals, one involving just the root $\varepsilon_{R}$ and the other the roots $\varepsilon_{1}, \ldots, \varepsilon_{R-1}$. Thus the set $I$ consisting of all indices $i<R$ for which $n_{i R} \neq 0$ is nonempty. By Proposition $1, n_{i R} \neq 0$ implies

$$
n_{i} \geq n_{R}, \quad n_{h i}=n_{h R}, \quad i \in I, \quad h<i .
$$

For $h<i$ with nonzero $n_{h R}, n_{i R}$, we have $n_{h i}=n_{h R} \neq 0$ and Proposition 1 gives

$$
n_{h i}=n_{h R} \geq n_{i R}, \quad h, i \in I, \quad h<i .
$$

Let $I$ be the complement of $I$ in $\{1, \ldots, R-1\}$. We will use Greek letters $\alpha, \beta$ for indices in $\bar{I}$ and Roman letters $h, i, j$ for indices in $I$. From (7), we have

$$
n_{\alpha i}=0, \quad \alpha \in \bar{I}, \quad i \in I, \quad \alpha<i .
$$


Fix an index $i \in I$. Using (5), (6), and (9), the relation $\omega\left(X_{i}\right) \leq \omega\left(X_{R}\right)$ becomes

$$
\frac{1}{2} n_{i}+\sum_{h<i} n_{h i}+\sum_{\beta>i} n_{i \beta}+\sum_{j>i} n_{i j}+n_{i R} \leq \frac{1}{2} n_{R}+\sum_{h<R} n_{h R}
$$

or, using the second equation in (7),

$$
\frac{1}{2}\left(n_{i}-n_{R}\right)+\sum_{\beta>i} n_{i \beta}+\sum_{j>i}\left(n_{i j}-n_{j R}\right) \leq 0
$$

where each term on the left is nonnegative by (7) and (8). Thus we have

$$
\begin{aligned}
& \quad n_{i \beta}=0, \quad \beta \in \bar{I}, \quad i \in I, \quad i<\beta, \\
& n_{i}=n_{R}, \quad n_{i j}=n_{j R}, \quad i, j \in I, \quad i<j .
\end{aligned}
$$

If $\bar{I}$ is nonempty, (9) and (10) imply that $\underline{s}$ decomposes into two $j$-invariant ideals, one involving the roots $\varepsilon_{\alpha}, \alpha \in \bar{I}$, the other involving the roots $\varepsilon_{i}, i \in I \cup\{R\}$. This proves our first claim.

To complete the proof of the proposition, set $a=n_{R}, b=n_{1 R}$. Since $I=\{1, \ldots$, $R-1\},(11)$ shows $n_{i}=a$ for all $i \leq R$ and (8) and (11) show that $n_{i R}=n_{i j}=n_{j R}$ for all $i<j<R$ which clearly implies $n_{i j}=b$ for all $i<j \leq R$.

Note that for $R>1$, we showed $b>0$ but $a=0$ is always possible.

2. This section relies on the theory of homogeneous cones as developed by Köcher, Rothaus, Vinberg, and Dorfmeister among others. It will be easiest for us to use Vinberg $[16,17]$ as a reference although some of this material was found independently by Köcher and Rothaus [8-10, 14]. Also, since the results we need are expressed in terms of a homogeneous Siegel domain and its cone, we shall find it convenient to fix a Siegel domain $D$ corresponding to the normal $j$-algebra $(\underline{s}, j)$ under consideration. For this purpose, we first recall a construction of Pyatetskii-Shapiro [13].

Let $(\underline{s}, j)$ be a normal $j$-algebra and let

$$
\underline{L}=\sum \underline{\underline{n}}_{\varepsilon_{k}}+\sum_{k<l} \underline{n}_{\left(\varepsilon_{k}+\varepsilon_{l}\right) / 2}, \quad \underline{U}=\sum \underline{n}_{\varepsilon_{k} / 2} .
$$

Let $E=\Sigma X_{k}$. Since $j \underline{L}$ is a subalgebra and $\underline{L}$ is an abelian ideal, we have for each $j X \in j \underline{L}$, a linear transformation $e^{\text {adj } X}$ on $L$. This gives us a group of linear transformations, which we shall denote by $\exp \operatorname{ad}_{L} j \underline{L}$, with Lie algebra $j \underline{L}$. Letting $\Omega$ be the orbit of $E$ under this group of transformations, one can show [13, pp. 66-73] $\Omega$ is a regular cone in $\underline{L}$ and that

$$
D=\left\{(X+i Y, U): X, Y \in \underline{L}, U \in \underline{U}, Y-\frac{1}{4}[j U, U] \in \Omega\right\}
$$

is a homogeneous Siegel domain on which $S$ acts simply transitively by affine transformations. Further, the diffeomorphism $S \ni g \mapsto g(i E, 0) \in D$ is biholomorphic with respect to the left invariant complex structure induced by $j$ on $S$ and the natural complex structure induced on $D$ as an open set in $\underline{L}^{C} \oplus \underline{U}$ (the complex vector space structure on $\underline{U}$ is given by $j$ ). As explained earlier, the Bergman metric on $D$ then corresponds to a left invariant metric on $S$ with $\omega$ given by (6). There are two principal results we need. First, we must compute the $T$-algebra associated to the cone $\Omega$ which 
in turn requires the computation of the clan (left-symmetric) algebra of $\Omega$. (Compare with [15] which computes the differential geometry of the cone starting from the $T$-algebra.) Second, we need to show that the connectedness algebra structure on $\underline{L}$ agrees with the algebra structure defined by Dorfmeister $[5,6]$ under the assumption that the dimensions of the root spaces behave as in Proposition 2.

2.1. The T-algebra of $\Omega$. According to [16, p. 360], one starts the construction of the clan of $\Omega$ by taking a simply transitive triangular affine group acting on $\Omega$ and a base point in $\Omega$. We will take exp $\operatorname{ad}_{L} j \underline{L}$ and $E$ for this purpose. For any $Y \in \Omega$, we identify as usual the tangent space of $\Omega$ at $Y$ with the ambient vector space $L$. Then any $j X \in j \underline{L}$, thought of as the Lie algebra of $\exp \operatorname{ad}_{L} j \underline{L}$, defines a vector field $(j X)^{*}$ on $\Omega$ by

$$
(j X)_{Y}^{*}=\left.\frac{d}{d t}\right|_{t=0}(\exp \operatorname{ad} t j X)(Y)=[j X, Y] .
$$

In particular, this gives a map from the Lie algebra of the transformation group to the tangent space of the cone at $E$ by

$$
j X \mapsto(j X)_{E}^{*}=[j X, E]=j[X, E]-[X, j E]-j[j X, j E]=X
$$

where here we have used the relations $[\underline{L}, \underline{L}]=0$ and $\operatorname{ad} j E|\underline{L}=I, \operatorname{ad} j E| j \underline{L}=0$. In the notation of [16], this means that $D_{X}=L_{X}=j X$. Then one defines a product $\triangle$ on $\underline{L}$ so that for $X, Y \in \underline{L}$,

$$
X \triangle Y=\left(L_{X}\right)_{Y}^{*}=(j X)_{Y}^{*}=[j X, Y] .
$$

The algebra $(L, \Delta)$ is called the clan or left symmetric algebra of $\Omega$. Clearly $X_{k} \Delta$ $X_{l}=\left[j X_{k}, X_{l}\right]=\delta_{k l} X_{l}$ which means that each $X_{k}$ is an idempotent of the clan. It is easy to check that the decomposition

$$
\underline{L}=\sum_{k \leq l} \underline{n}_{\left(\varepsilon_{k}+\varepsilon_{l}\right) / 2}
$$

is a normal decomposition of the clan in the sense of [16, p. 373].

According to [16, p. 397], one now constructs the $T$-algebra corresponding to $\Omega$ as follows. For each space $\underline{n}_{\left(\varepsilon_{k}+\varepsilon_{l}\right) / 2}$ with $k<l$ in the normal decomposition of the clan, pick an isomorphic vector space $A_{k l}$ and a fixed isomorphism $X \mapsto \hat{X}$ of $\underline{n}_{\left(\varepsilon_{k}+\varepsilon_{l}\right) / 2}$ onto $A_{k l}$. For any $X \in \underline{L}$, let $X_{k l}$ be the component of $X$ in $\underline{n}_{\left(\varepsilon_{k}+\varepsilon_{l}\right) / 2}$. Let $N$ be the direct sum of the $A_{k l}, k<l$, and define a product in $N$ by

$$
\hat{X} \hat{Y}=\sum_{k<l<m}\left(X_{k l} \Delta Y_{l m}\right)^{\wedge}
$$

and an inner product by

$$
(\hat{X}, \hat{Y})=\frac{1}{2} \operatorname{Tr}_{L}(Z \mapsto(X \triangle Y) \triangle Z) .
$$

$N$ with this grading, product, and inner product is called an $N$-algebra, which is the nilpotent part of the desired $T$-algebra $A . A$ itself is a graded algebra $\sum A_{k l}$ where, for $k<l, A_{k l}$ is as already constructed and $N$ is a subalgebra. In particular, one has

$$
\operatorname{dim} A_{k l}=\operatorname{dim} \underline{\underline{n}}_{\left(\varepsilon_{\boldsymbol{k}}+\varepsilon_{l}\right) / 2} \text { for } k \neq l .
$$


Since this is the only relation we need, we will not complete the construction of the algebra structure.

2.2. The connectedness algebra of $\Omega$. Let $\phi$ be the characteristic function of the cone $\Omega[16$, p. 347] (see also the $M$-function, $[8$, p. 583]) which we may normalize so that $\phi(E)=1$. Let $G(\Omega)=\{g \in G L(\underline{L}): g(\Omega)=\Omega\}$. The only property of $\phi$ we need is that

$$
\phi(g(E))=(\operatorname{det} g)^{-1} \text { for } g \in G(\Omega) \text {. }
$$

Now the canonical Siegel domain $D$ corresponding to the normal $j$-algebra $(\underline{s}, j)$ is determined by the cone $\Omega$ and the $\Omega$-Hermitian function $F: \underline{U} \times \underline{U} \rightarrow \underline{L}^{\mathbf{C}}$ defined by

$$
F(U, V)=\frac{1}{4}([j U, V]+i[U, V]) .
$$

Let $G(\Omega, F)=\{g \in G(\Omega): \exists \hat{g} \in G L(\underline{U}, \mathbf{C})$ with $g(F(U, V))=F(\hat{g} U, \hat{g} V)$ for all $U$, $V \in \underline{U}\}$.

Let $B$ denote the Bergman kernel function of $D$. Then $[5,7,12]$ there is a holomorphic function $\eta$ on $\Omega+i \underline{L}$ such that

(14) $B\left(\left(Z_{1}, U_{1}\right),\left(Z_{2}, U_{2}\right)\right)=\eta\left(\frac{1}{2 i}\left(Z_{1}-\bar{Z}_{2}\right)-F\left(U_{1}, U_{2}\right)\right), \quad Z_{i} \in \underline{L} \mathbf{C}, \quad U_{i} \in \underline{U}$.

The basic property we need about $\eta$ is

$$
\eta(g(E))=(\operatorname{det} g)^{-2}\left|\operatorname{det}_{\mathbf{c}} \hat{g}\right|^{-2} \eta(E) \quad \text { for } g \in G(\Omega, F) .
$$

We emphasize that $\hat{g}$ is a complex linear transformation of the complex vector space $\underline{U}$ and the determinant is taken accordingly.

Fix any element $X \in \underline{L}$ and set

$$
g=e^{\operatorname{ad}_{l} j X}, \quad \hat{g}=e^{\operatorname{ad}_{y} j X} .
$$

Then $g$ is in $G(\Omega, F)$ (with $\hat{g}$ satisfying the required relation with $F$ ) and the set of all such transformations forms a subgroup of $G(\Omega, F)$ which acts transitively on $\Omega$ by definition. Let $\alpha: j \underline{L} \rightarrow \underline{a}$ denote projection with respect to the root space decomposition. Since

$$
\left\langle\left[\underline{n}_{\left(\varepsilon_{k}-\varepsilon_{l}\right) / 2}, \underline{n}_{\varepsilon_{m} / 2}\right], \underline{n}_{\varepsilon_{m} / 2}\right\rangle=0
$$

we see that

$$
\operatorname{Tr} \operatorname{ad}_{U} j X=\operatorname{Tr} \operatorname{ad}_{U} \alpha(j X) .
$$

Similarly

$$
\left\langle\left[\underline{n}_{\left(\varepsilon_{k}-\varepsilon_{l}\right) / 2}, \underline{n}_{\left(\varepsilon_{m}+\varepsilon_{n}\right) / 2}\right], \underline{n}_{\left(\varepsilon_{m}+\varepsilon_{n}\right) / 2}\right\rangle=\left\langle\left[\underline{n}_{\left(\varepsilon_{k}-\varepsilon_{l}\right) / 2}, \underline{n}_{\varepsilon_{m}}\right], \underline{n}_{\varepsilon_{m}}\right\rangle=0
$$

so

$$
\operatorname{Tr} \operatorname{ad}_{L} j X=\operatorname{Tr} \operatorname{ad}_{L} \alpha(j X) .
$$

But, for $H \in \underline{a}$, one computes

$$
\operatorname{Tr} \operatorname{ad}_{U} H=\sum \frac{1}{2} \varepsilon_{k}(H) \operatorname{dim} \underline{\underline{n}}_{\varepsilon_{k} / 2},
$$




$$
\operatorname{Tr} \operatorname{ad}_{L} H=\sum \varepsilon_{k}(H)+\sum_{k<l} \frac{1}{2}\left(\varepsilon_{k}+\varepsilon_{l}\right)(H) \operatorname{dim} \underline{n}_{\left(\varepsilon_{k}+\varepsilon_{l}\right) / 2}
$$

From now on, we assume that

$$
\operatorname{dim} \underline{n}_{\varepsilon_{i} / 2}=a, \quad 1 \leq i \leq R ; \quad \operatorname{dim} \underline{n}_{\left(\varepsilon_{k}+\varepsilon_{l}\right) / 2}=b, \quad 1 \leq k<l \leq R,
$$

for some constants $a$ and $b$. Then for $H \in \underline{a},(19)$ and (20) become

$$
\begin{gathered}
\operatorname{Tr} \operatorname{ad}_{U} H=\frac{1}{2} a \sum \varepsilon_{k}(H), \\
\operatorname{Tr} \operatorname{ad}_{L} H=\left(1+\frac{1}{2} b(R-1)\right) \sum \varepsilon_{k}(H) .
\end{gathered}
$$

Combining (16)-(18), (22), and (23) (and recalling that $a=n_{i}$ is real dimension), we have

$$
\begin{gathered}
\operatorname{det} g=e^{\operatorname{Tr}_{\mathrm{rad}} j X}=\left(e^{\Sigma \varepsilon_{k}(\alpha(X))}\right)^{(1+b(R-1) / 2)}, \\
\left|\operatorname{det}_{\mathbf{c}} \hat{g}\right|^{2}=\operatorname{det} \hat{g}=e^{\operatorname{Tr} \operatorname{ad}_{U} j X}=\left(e^{\Sigma \varepsilon_{k}(\alpha(X))}\right)^{a / 2}
\end{gathered}
$$

Thus we have

$$
\eta=\eta(E) \phi^{c} \text { for some constant } c .
$$

Now one defines $[16$, p. $350 ; 8$, p. 585] a Riemannian metric (,) on the cone $\Omega$ so that in a Euclidean coordinate system $x^{1}, \ldots, x^{n}$ on the vector space $L$, the metric tensor is given by

$$
g_{i j}=\frac{\partial^{2}}{\partial x_{i} \partial x_{j}} \ln \phi
$$

Identify $\underline{L}$ with the tangent space to $\underline{L}$ at $E$ and for each $Y \in \underline{L}$, let $Y^{0}$ denote the translation invariant vector field on $\underline{L}$ which agrees with $Y$ at $E$. Then [16, p. 350; 10, p. 194] one defines a product on $\underline{L}$, denoted $\square$, by

$$
Y_{1} \square Y_{2}=-\left(\tilde{\nabla}_{Y_{1}^{0}} Y_{2}^{0}\right)_{E}
$$

where $\tilde{\nabla}$ is the Riemannian connection for (,). $L$ with the product $\square$ is called by Vinberg the connectedness algebra of $\Omega$ (perhaps connection algebra would be a better term). For $Y_{1}, Y_{2}, Y_{3} \in \underline{L}$, one has $\left[Y_{i}^{0}, Y_{j}^{0}\right]=0$ and

$$
\begin{aligned}
-2\left(Y_{1} \square Y_{2}, Y_{3}\right)_{E} & =2\left(\tilde{\nabla}_{Y_{1}^{0}} Y_{2}^{0}, Y_{3}^{0}\right)_{E} \\
& =\left(Y_{1}^{0} \cdot\left(Y_{2}^{0}, Y_{3}^{0}\right)-Y_{3}^{0} \cdot\left(Y_{1}^{0}, Y_{2}^{0}\right)+Y_{2}^{0} \cdot\left(Y_{1}^{0}, Y_{3}^{0}\right)\right)_{E} \\
& =\left(\left(Y_{1}^{0} \cdot Y_{2}^{0} \cdot Y_{3}^{0}-Y_{3}^{0} \cdot Y_{1}^{0} \cdot Y_{2}^{0}+Y_{2}^{0} \cdot Y_{1}^{0} \cdot Y_{3}^{0}\right) \cdot \ln \phi\right)_{E} \\
& =\left(Y_{1}^{0} \cdot Y_{2}^{0} \cdot Y_{3}^{0} \cdot \ln \phi\right)_{E} .
\end{aligned}
$$

Now we want to compare this result with the product defined in $[5, \mathrm{p} .14 ; 6, \mathrm{p}$. 539]. First define a bilinear form $\sigma$ on $\underline{L}$ by

$$
\sigma\left(Y_{1}, Y_{2}\right)=\left(Y_{1}^{0} \cdot Y_{2}^{0} \cdot \ln \eta\right)_{E}
$$

Notice that under assumption (21), we have $\sigma=c(,)_{E}$ by (24). The new product is 
defined by the requirement that

$$
\sigma\left(Y_{1} Y_{2}, Y_{3}\right)=-\frac{1}{2}\left(Y_{1}^{0} \cdot Y_{2} \cdot Y_{3}^{0} \cdot \ln \eta\right)_{E} .
$$

Comparing (27) with (28), it is clear that these products agree under assumption (21). Remark. The product defined by (28) is exactly the product defined by (26) when $D$ is the tube domain over $\Omega$ since then the metric defined in (25) is just a constant multiple of the Bergman metric. When $D$ is not a tube domain, the Bergman metric on $D$ induces a metric on the tube domain $D^{\prime}$ considered as a submanifold of $D$. This induced metric is generally quite different from the Bergman metric of $D^{\prime}$. The point here is that under assumption (21), these two metrics on the tube domain $D^{\prime}$ do agree up to constant multiple. Comparing results of [3 and 21], one can also see the product defined by (28) agrees with one defined by Satake in the quasi-symmetric case.

\subsection{Conclusions.}

Proposition 3. The domain $D$ corresponding to the irreducible normal j-algebra $(\underline{s}, j)$ is quasi-symmetric if and only if the dimensions of the root spaces of $\underline{s}$ satisfy $(21)$. (Compare with Zelow (Lundquist) [21].)

Proof. The notion of quasi-symmetic as used here was introduced by Satake. We will use the formulation of Dorfmeister [5, 6].

Suppose (21) holds. From (12), we know the $T$-algebra of the associated cone $\Omega$ satisfies $\operatorname{dim} A_{k l}=b$ for all $k \neq l$. According to [17, p. 73], this means $\Omega$ is self-dual with respect to some inner product on $\underline{L}$. Again according to [17], this implies that the connectedness algebra $(\underline{L}, \square)$ is Jordan. By the results of $(2.2)$, the connectedness algebra structure on $L$ agrees with that defined in [5, 6] which is therefore also Jordan. This implies the domain is quasi-symmetric.

Suppose $D$ is quasi-symmetric and irreducible. The cone is then irreducible and self-dual so the result of Vinberg says $n_{k l}=b$ for $k<l$. But for a quasi-symmetic irreducible domain, one knows $\omega\left(X_{k}\right)=\omega\left(X_{l}\right)$ where $\omega$ gives the Bergman metric $[3,21]$. By (6), we have $n_{i}=a$ for all $i$.

THEOREM. Suppose the domain D has nonpositive sectional curvatures in the Bergman metric. Then $D$ is symmetric.

Proof. As explained earlier, we may assume $D$ is irreducible. By Proposition 2, assumption (21) holds. By Proposition 3, the domain is quasi-symmetric. By [3], a quasi-symmetric domain with nonpositive sectional curvature in the Bergman metric is symmetric.

REMARKS. We would like to close with what we feel are some remaining interesting open questions concerning the curvature of homogeneous domains.

(1) How does one characterize the homogeneous Siegel domains whose holomorphic sectional curvatures in the Bergman metric are all nonpositive? That this is not true for all domains seems actually to first occur in [11]; see also [2]. From [19, 20], one knows this holds for all quasi-symmetric domains, but from [2, 3] one also knows there are non-quasi-symmetric domains with this property. 
(2) According to [3] and (1.2) there are some conditions on the normal $j$-algebra $(\underline{s}, j)$ which guarantee that there is no choice of $\omega$ giving a Kähler metric on the associated domain $D$ with nonpositive sectional curvature. On the other hand, there are normal $j$-algebras corresponding to nonsymmetric domains in which such a choice of $\omega$ (and corresponding Kähler metric) does exist. Can one characterize the homogeneous Siegel domains for which there exists a transitive group $G$ of biholomorphic transformations and a $G$ invariant Kähler metric with nonpositive sectional curvature? In such a situation, how big is the isometry group?

\section{BIBLIOGRAPHY}

1. J. E. D'Atri, The curvature of homogeneous Siegel domains, J. Differential Geom. 15 (1980), 61-70.

2. - Holomorphic sectional curvatures of bounded homogeneous domains and related questions, Trans. Amer. Math. Soc. 256 (1979), $405-413$.

3. - Sectional curvatures and quasi-symmetric domains, J. Differential Geom. 16(1981), 11-18.

4. J. Dorfmeister, Inductive construction of homogeneous cones, Trans. Amer. Math. Soc. 252 (1979), 321-349.

5. - Homogene Siegel-Gebiete, Habilitationsschrift, Westfälischen Wilhelms-Universität, Münster, 1979.

6 , Quasisymmetric Siegel domains and the automorphisms of homogeneous Siegel domains, Amer. J. Math. 102 (1980), 537-563.

7. S. G. Gindikin, Analysis in homogeneous domains, Uspehi Mat. Nauk 19 (1964), 3-92; English transl., Russian Math. Surveys 19 (1964), 1-89.

8. M. Köcher, Positivitäts bereiche im $\mathbf{R}^{n}$, Amer. J. Math. 79 (1957), 575-596.

9. - Analysis in reelen Jordan Algebren, Nachr. Akad. Wiss. Göttingen Math.-Phys. K1. IIa (1958), 67-74.

10. —, Die Geodätischen von Positivitätsbereichen, Math. Ann. 135 (1958), 192-202.

11. K. H. Look (Lu Chi-Keng) and Hsu I-chau (Hsü I-chao), A note on transitive domains, Acta Math. Sinica 11 (1961), 11-23; English transl., Chinese J. Math. 2 (1962), 11-26.

12. S. Murakami, On automorphisms of Siegel domains, Lecture Notes in Math., vol. 286, SpringerVerlag, Berlin and New York, 1971.

13. I. I. Pyatetskii-Shapiro, Automorphic functions and the geometry of classical domains, Gordon \& Breach, New York, 1969.

14. O. S. Rothaus, Domains of positivity, Abh. Math. Sem. Univ. Hamburg 24 (1960), 189-235.

15. T. Tsuji, A characterization of homogeneous self-dual cones, preprint, 1981.

16. E. B. Vinberg, The theory of convex homogeneous cones, English transl., Trans. Moscow Math. Soc. 12 (1963), 340-403.

17. - The structure of the group of automorphisms of a homogeneous convex cone, Trans. Moscow Math. Soc. 13 (1965), 63-93.

18. R. Zelow (Lundquist), On the geometry of some Siegel domains, Nagoya Math. J. 73 (1979), 175-195.

19. - Curvature of quasi-symmetric Siegel domains, J. Differential Geom. 14 (1979), 629-655.

20. - Holomorphic sectional curvature of quasi-symmetric domains, Proc. Amer. Math. Soc. 76 (1979), 299-301.

21. —, Quasi-symmetric domains and j-algebras, preprint, Oslo, 1979.

Department of Mathematics, Rutgers University, New Brunswick, New Jersey 08903

I. M. A. F., Universidad Nacional de Cordoba, Cordoba 5000, Argenting 